access to specialist palliative care services and poor overall quality of care in the last three months of life.

Aim To describe the inpatient population of a hospice ward and to explore the relationship between level of deprivation and the prevalence of co-morbidities.

Methods Data were collected from patient notes, nursing documentation and computer systems for 30 patients (total number of beds) over 1 week. Scottish Index of Multiple Deprivation quintiles (SIMD 1 - most deprived; SIMD 5 - least deprived) were assigned to assess level of deprivation based on patient postcode. Data were analysed using t-test on Graphpad PRISM.

Findings The sample comprised of 15 female and 15 male patients ranging in age from 56-88 years. Eighteen had been admitted from home, 11 from hospital and one from the hospice outpatient clinic. Primary disease was more commonly malignant than non-malignant (26:4 respectively). Fourteen were from SIMD quintile 1 (most deprived), but only two were from SIMD 5 (least deprived). Patients from areas of high deprivation had nearly double the number of admissions to hospital in the preceding 12 months (SIMD 1-2 mean 3.7 (95\% CI: 2.4-5.1); SIMD 3 and above 2.1 (1.4-2.7) $\mathrm{P}=0.02$ ). The number of co-morbidities ranged from a single disease to nine; there was no correlation with SIMD quintile.

Conclusion The patients captured in this study were predominately from more deprived areas, which reflects the local population. Importantly, these patients had significantly more admissions to hospital compared with patients from less deprived areas. More research is required to understand the reasons for this, including the extent to which these admissions are occurring out of hours, and to determine the level of need for patients and families living and dying in deprived areas.

\section{P-97 HOSPICE WORKING IN PARTNERSHIP WITH THE HOMELESS COMMUNITY}

Sue Scully. St Barnabas Hospice Trust, Lincoln, UK

\subsection{6/bmjspcare-2016-001245.120}

Hospice day therapy staff have been engaging with the homeless community via local services who support them, enabling those who have a life limiting condition(s) the equal opportunity to take control of their lives through the support of our specialist palliative rehabilitation service regardless of their homeless status.

Our aim is to optimise a person's physical function and emotional wellbeing, consistent with their choice, goals and priorities. We work together with patients and those who are important to them using our expertise, to equip them with new tools to maximise wellbeing.

The majority of patients that we care for who are part of this vulnerable and marginalised community, usually present with underlying mental health illnesses and may have alcohol and/or drug addictions. They experience significant ill health and the average age of death is 47 years.

It can be difficult for these people to engage with our services, due to their transient life styles and their psychological and emotional problems. The holistic assessment can be completed at point of contact or at the Day Therapy Centre. If patients do not attend with consent we continue to have contact with their support worker, housing officer.

Other members of staff and students are invited to attend from the Trust. This highlights to other professionals the ongoing difficulties that these patients have and proves that as a Hospice
Trust we are committed to making our service accessible, demonstrating the diverse groups that we support.

With monthly engagement with the services we have forged strong professional relationships with support workers/housing officers and managers and referrals are starting to increase.

Statistics Mean = May 2015-May 2016 monthly contact/discussion with 10 service users

Service users who accessed the centre during this period $=5$

Service users who engaged at point of contact $=4$.

\section{P-98 WE HEAR NOW AND THEN: A SECONDARY QUALITATIVE ANALYSIS OF INNER CITY LONDON MINORITIES' EXPERIENCES WITH HOSPICE CARE}

Munikumar Ramasamy Venkatasalu. Universiti Brunei Darussalam, Gadong, Brunei Darussalam

\subsection{6/bmjspcare-2016-001245.121}

Background Hospices undertake diverse social inclusion strategies to enable hospice access for minority population. Yet, utilisation and access to hospice care among British minorities remain scanty.

Objective To explore perspectives of older British South Asians on use of hospices at the end-of-life.

Design A constructivist grounded theory study.

Methods A secondary qualitative data analysis was taken on views of 55 older British South Asian participants, recruited using purposive sampling. Participants were approached through their community leaders and at open meetings of 11 local South Asian community groups of East London, England. Five focus groups and 29 semi-structured interviews were conducted. Thematic analysis was taken.

Results Three themes arrived: misconceptions, expectations and strategies. Participants described misconceptions related to hospice access including payable hospices and image of nursing home care as hospice care. Analysis also shows that lack of experience often created such misconceptions. Expectations include change in physical and emotional surroundings and enabling home care hospice services. Participants reported various ethniccentred inclusion strategies to promote hospice concept among this minority population.

Conclusion Current social inclusion strategies of hospice seems to promote the spread of hospice concept among these older inner city minorities. However, access and utilisation of hospice services remain 'distant' to real usage. Future strategies should focus microlevel social interventions and mass media based interventions to promote hospice usage among this population.

\section{P-99 A CULTURALLY INCLUSIVE, BAME-COMMUNITY-LED SERVICE FOR THOSE REACHING THE END OF LIFE}

Muhammad Abdullah, Caroline Mawer, Rupina Begum, Abu Mumin. Edencare UK, London, UK

\subsection{6/bmjspcare-2016-001245.122}

Background People from BAME communities are known to have specific palliative care needs. Different cultural approaches may have substantial impacts both within families, and on service use. Despite this, services are often provided 'for', rather than 'developed with' Muslims. In response to this, Muslim community 